\title{
AN ANALYSIS OF SKIN APPENDAGEAL TUMORS IN SOUTH INDIA.
}

S. Sri Gayathri, Ezhilvizhi Alavandar, S. Ashok Kumar

1. Assistant Professor, Department of Pathology. SRM Medical College \& Research Centre.

2. Professor, Department of Pathology. Govt Kilpauk Medical College.

3. Assistant Professor, Department of Pathology. Chengelpet Medical College.

\section{CORRESPONDING AUTHOR}

Dr. S. Sri Gayathri, 602 B Block, SRM Medical Staff Quarters,

SRM Medical College, Potheri

Kanchipuram District, Tamilnadu - 603203.

E-mail: ssriga@yahoo.com

Ph: 00919841129271

ABSTRACT: BACK GROUND: Cutaneous appendageal tumours are a large diverse group of tumours that are commonly classified according to their state of appendageal differentiationeccrine, apocrine, follicular and sebaceous. MATERIALS AND METHODS: This is a retrospective study that reviewed the clinical records of patients from the Department of pathology, Govt Kilpauk medical college who were diagnosed with skin tumours by histopathology for a period of three years. Afterwards, these cases were, reviewed diagnoses were recorded and classified and analysed according to the patient's age, gender, and localization, occupation, exposure to sunlight and clinical presentation. RESULTS: This descriptive study was an analysis of 29 adnexal tumors with regard to age, clinical presentation, occupation and its correlation with histologic diagnosis. Benign tumors were common and composed of $74 \%$ and malignant tumors were $26 \%$. . Mean age of presentation of adnexal tumors in this study was 35.2 years with female predominance. 15 of these tumours were derived from eccrine and apocrine glands, 11 of them showed follicular differentiation, and 3 of them showed sebaceous differentiation. They showed a maximum occurrence in face as nodular and papular lesions. Commonest lesion was Trichoepithelioma. CONCLUSION: Adnexal skin tumors have distinct histological patterns which differentiates them from other cutaneous tumors. Clinical diagnosis of adnexal tumors is extremely difficult because various adnexal tumors have similar clinical presentation and histopathological examination becomes essential in diagnosis

KEY WORDS: Appendageal, apocrine glands, eccrine, follicular differentiation.

INTRODUCTION: Cutaneous appendageal tumours are a large diverse group of tumours that are commonly classified according to their state of appendageal differentiation- eccrine, apocrine, follicular and sebaceous ${ }^{1,2,3}$. These tumours generally behave in a benign manner, but malignant types exist. ${ }^{4}$ Their detailed morphological classification is difficult because of the variety of tissue elements and patterns seen. In our present study we have done a histopathologic analysis and clinicopathologic correlation of cutaneous adnexal tumors.

MATERIAL AND METHODS: We have reviewed the clinical records of patients from the Department of pathology, Govt Kilpauk medical college who were diagnosed with skin tumours by histopathology for a period of three years. This is a cross sectional descriptive study of appendageal tumors in department of Pathology. All biopsies were taken from grossly characteristic areas. Multiple biopsies were advised when lesions present in differing forms and 
stages. Biopsies received in 10\% neutral buffered formalin, processed in routine manner and stained with Hematoxylin and eosin stains. Special stains like PAS and VanGieson were done when needed. An analysis of skin adnexal tumors, their age, site and clinical presentation was done and correlated with histologic diagnosis, and occupational sunlight exposure .

RESULTS: The study was analysis of 29 adnexal tumors. 27 of them are benign and 2 are malignant. 15 of these tumours were derived from eccrine and apocrine glands, 11 of them showed follicular differentiation, and 3 of them showed sebaceous differentiation. As given in Table 1, Male: female ratio is 1:1.6 with female predominance. Mean age of presentation of adnexal tumors in this study is 35.2 years. 52\% of adnexal tumors show eccrine and apocrine differentiation and $48 \%$ of them show pilosabeceous differentiation. Commonest adnexal tumor in this study is Trichoepithelioma. Appendageal tumours present mostly as single lesions. They show a maximum occurrence in face as nodular and papular lesions. Tumours of follicular differentiation are multiple papular lesions more often seen in face. The malignant Appendageal tumours are two in number with follicular differentiation.

All these tumors did not show any occupational exposure to excessive sunlight.

DISCUSSION: Cutaneous appendageal tumours are a large diverse group of tumours that are commonly classified according to their state of appendageal differentiation- eccrine, apocrine, follicular and sebaceous. These tumours generally behave in a benign manner, but malignant types exist. 29 appendageal tumours were observed in the study. Of which 15 were sweat gland tumours, 3 were sebaceous gland tumours and 11 showed follicular differentiations.

(I)SWEAT GLAND TUMOURS HIDROCYSTOMA: One case of hidrocystoma in the study showed single cystic cavity with two layers of small cuboidal cells. It presented as solitary bluish cystic nodule in neck region. Bluish colour is due to presence of lipofuschin, melanin, hemosiderin and Tyndall effect.

CHONDROID SYRINGOMA: Mixed tumor of skin is the term introduced by Hirsch and Helwig in $1961^{5}$ has been replaced by the term Chondroid Syringoma. 2 cases observed in the study were firm intradermal nodules seen in face. Hirsch.P. 5 observed the chondroid syringomas to occur more commonly in head and neck. Similar observations were observed in the study. Histologically, tubular lamina with two layers of epithelial cells in a chondroid matrix is noted. (Figure 1)

NODULAR HIDRADENOMA: 5 cases of nodular Hidradenoma observed in the present study were intradermal nodular lesions in head and neck region presenting in the age group of 30-40 years.

SPIRADENOMA: One case of Spiradenoma in the present study was a solitary intradermal nodule over the face. The tumor was highly cellular with sharply demarcated lobules containing intertwining cords of epithelial cells, surrounded by capsule f compressed connective tissue. Two types of cells were noted small basophilic cells at the periphery and large pale cells in the center. The much-appreciated histological clue the perivascular spaces also observed in the present study was first described by Van Den oord and Chris De wolf Peters in $1998^{6}$. It consists of variably sized spaces around one or more central blood vessels bordered at the periphery by 
a palisade of tumor cells and lined at both sides by basement membrane. The spaces are empty or filled by pink proteinaceous material.

SYRINGOCYSTADENOMA PAPILLIFERUM: 4 cases of Syringocystadenoma papilliferum observed were commonly found in the scalp and face. Histologically, epidermis showed cystic invaginations lined by keratinizing squamous cells in the upper part and papillary projections lined by two rows of epithelium in the lower part. The stroma was densely cellular with plasma cells. (Figure 2)

ECCRINE POROMA: One case of eccrine poroma observed in the study was seen as firm raised asymptomatic nodule in forearm. Histologically, the tumor grows downward into the dermis as broad anastomosing bands of epithelial cells that are smaller than keratinocytes.

(II)TUMOURS OF SEBACEOUS GLANDS: 4 cases of nevus sebaceous were seen in the scalp,(Figure 3) which is the common site. Histologically tumor showed proliferations of sebaceous glands, papillomatous epidermis and ectopic apocrine glands deep in the dermis. ${ }^{7}$

(III)TUMOURS WITH FOLLICULAR DIFFERENTIATION: Histopathologically 33\% of tumours show follicular differentiation, compared to $63.4 \%$ by Jayalakshmi et al ${ }^{4}$.

TRICHOEPITHELIOMA: 6 cases of Trichoepithelioma were observed in the study. All were multiple lesions in the form of skin colored papules mainly if the nose and nasolabial fold. Histologically, all of them showed horncyst surrounded by basophilic cells showing Trichilemmal keratinisation, except for one case where there was no horn cyst formation. Islands of basophilic cells show peripheral pallisading. Important histopathological differential diagnosis is Basal Cell Carcinoma and was differentiated by the absence of retraction artifact of stroma. Papillary mesenchymal bodies, amyloid deposits, mitosis and inflammatory granuloma are other features that distinguish trichoepithelioma from Basal cell Carcinoma.

PILOMATRICOMA (SYNONYM: CALCIFYING EPITHELIOMA OF MALHERBE): Pilomatricoma are benign tumours with differentiation towards hair matrix. ${ }^{8} 3$ case of Pilomatricoma observed in the study were found in face and upper extremities occurring more commonly in the age group of less than 20 years. Histologically, the tumor is composed of irregular shaped islands of two types of epithelial cells (basophilic cells and shadow cells) with calcification and foreign body reaction. Malignant transformation in Pilomatricoma was not observed in the present study.

One case of Proliferating trichilemmal tumour with malignant transformation observed in the study was seen in scalp as an elevated lobular mass with ulcerations. Histologically, the tumor was composed of multiple lobules of squamous epithelium with peripheral pallisading. The tumor showed extensive areas of trichilemmal keratinisation, tissue invasion and presence of severe atypia and giant nuclei.

TRICHOBLASTIC CARCINOMA: One case of Trichoblastic carcinoma was observed in the present study. Histologically, it was made of proliferation of hair germ cells in the form of islands of basaloid cells with peripheral pallisading, occupying the dermis and infiltration into 
subcutaneous fat ${ }^{9}$. They showed moderate degree of cytological atypia. It was differentiated from basal cell carcinoma by lack of clefting artifact, stromal edema and ulceration ${ }^{10}$.

CONCLUSION: Adnexal skin tumors have distinct histological patterns which differentiates them from other cutaneous tumors. They are commonly distributed in the head, neck and trunk. The commonest are benign variants from eccrine sweat gland origin and the malignant tumors are uncommon. Clinical diagnosis of adnexal tumors is extremely difficult because various adnexal tumors have similar clinical presentation and histopathological examination becomes essential in diagnosis. Since most of adnexal tumors are benign excision is curative.

\section{REFERENCES:}

1. Samaila M. Adnexal skin tumors in Zaria, Nigeria. Ann Afr Med [serial online] 2008 [cited 2012 Sep 21]; 7:6-10.

2. Nair PS . A clinicopathologic study of skin appendageal tumors.Indian J Dermatol Venereol Leprol. 2008 Sep-Oct; 74(5):550.

3. Yaqoob N, Ahmad Z, Muzaffar S. Spectrum of cutaneous appendage tumors at Aga Khan University Hospita. J Pak Med Assoc. 2003 Sep; 53(9):427-31.

4. Jayalakshmi, Looi LMi P. Cutaneous adnexal neoplasms in biopsy specimens processed in the Department of Pathology, University of Malaya. Ann Acad Med Singapore1996 July; 25(4):522-5.

5. Ken Hashimotos, Tumors of Skin Appendages in Fitzpatrick Dermatology in General Medicine, Fitzpatrick TB 5 th edn Mc Grawhill 1999; 890-914.

6. Joost J Van den Oord et al ; Perivascular Spaces in eccrine Spiradenoma ; A clue to histological Diagnosis; AmJ Dermatopath 1995, 17(3) 266-270.

7. Manonukul , Kajornvuthidej SJ Sebaceous neoplasms in Siriraj Hospital, Mahidol University: a 9-year-retrospective study. J Med Assoc Thai. 2010 Aug;93(8):978-91.

8. U. Jindal , R. Patel : Study Of Adnexal Tumors Of The Skin: A Three Year Study Of 25 Cases. The Internet Journal of Pathology. 2012 Volume 13 Number 3. DOI: 10.5580/2bf5[PUBMED]

9. Abanti Saha, Nilay K Das, Ramesh C Gharami, Satyendra N Chowdhury, and Pijush K Datta, Indian J Dermatol. 2011 Jan-Feb; 56(1): 33-36.

10. Philip E Heboit et al WHO Classification of Skin Tumors , Pathology and Genetics of Skin Tumors, IARC Press LYON 2006.

\section{TABLES}

Table 1: Analysis of 29 adnexal tumors

\begin{tabular}{|l|l|}
\hline Benign & $74 \%$ \\
\hline Malignant & $26 \%$ \\
\hline Male: female ratio & $1: 1.6$ \\
\hline Mean age & 35.2 years \\
\hline Eccrine and apocrine differentiation & $52 \%$ \\
\hline Pilosebaceous differentiation & $48 \%$ \\
\hline Commonest adnexal tumor & Trichoepithelioma \\
\hline
\end{tabular}




\section{ORIGINAL ARTICLE}

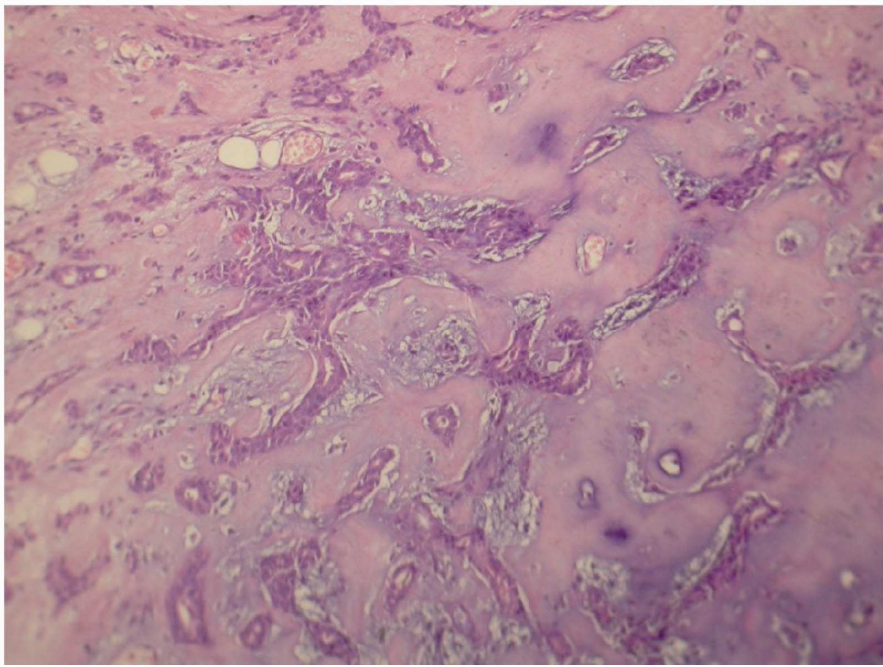

Figure1: Chondroid syringoma Cords of tumor cells in cartilage

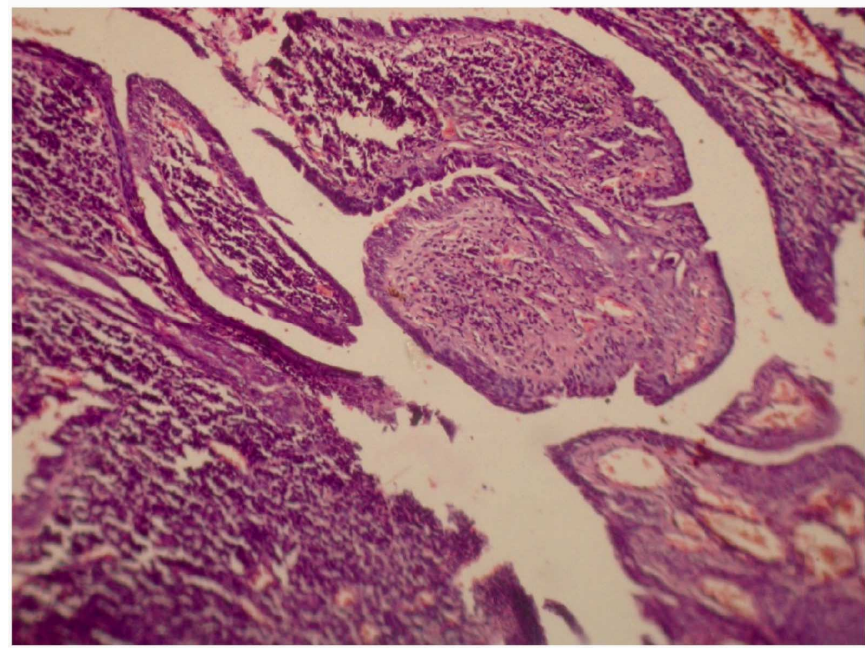

Figure 2: Syrigocystadenoma Papilliferum - Papillae lined by two layers of epithelial cells. 


\section{ORIGINAL ARTICLE}
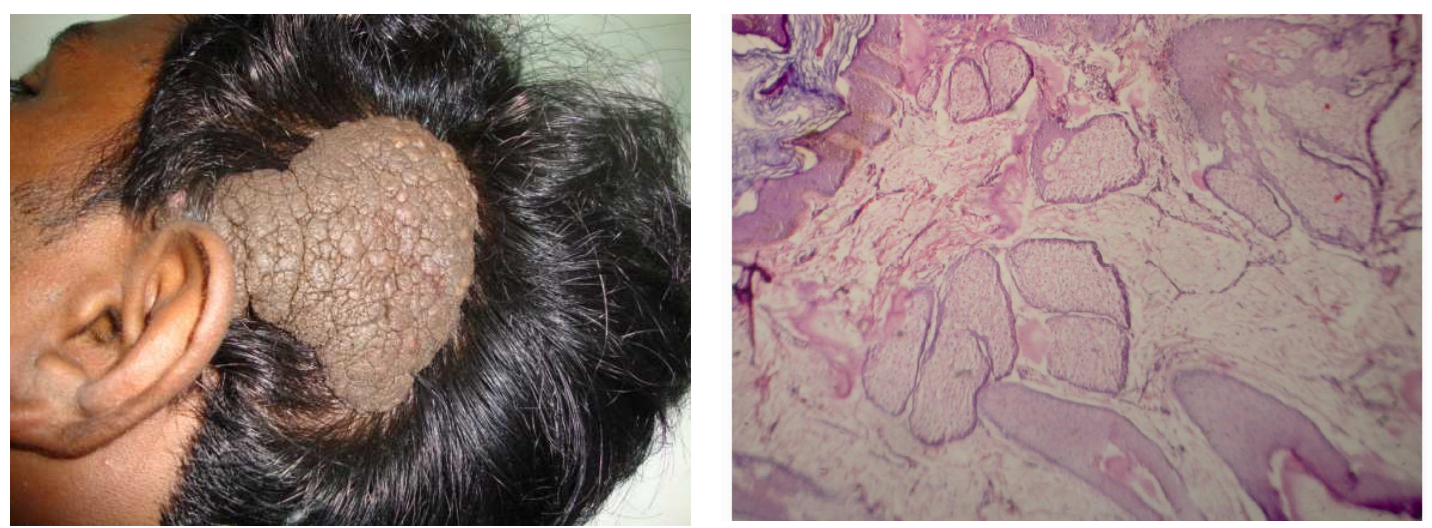

Figure 3: Nevus sebaceous A. Clinical picture, B. H \& E stain 10X.Lobules of sebaceous glands and hair follicles

Graph 1: Site distribution of adnexal tumors

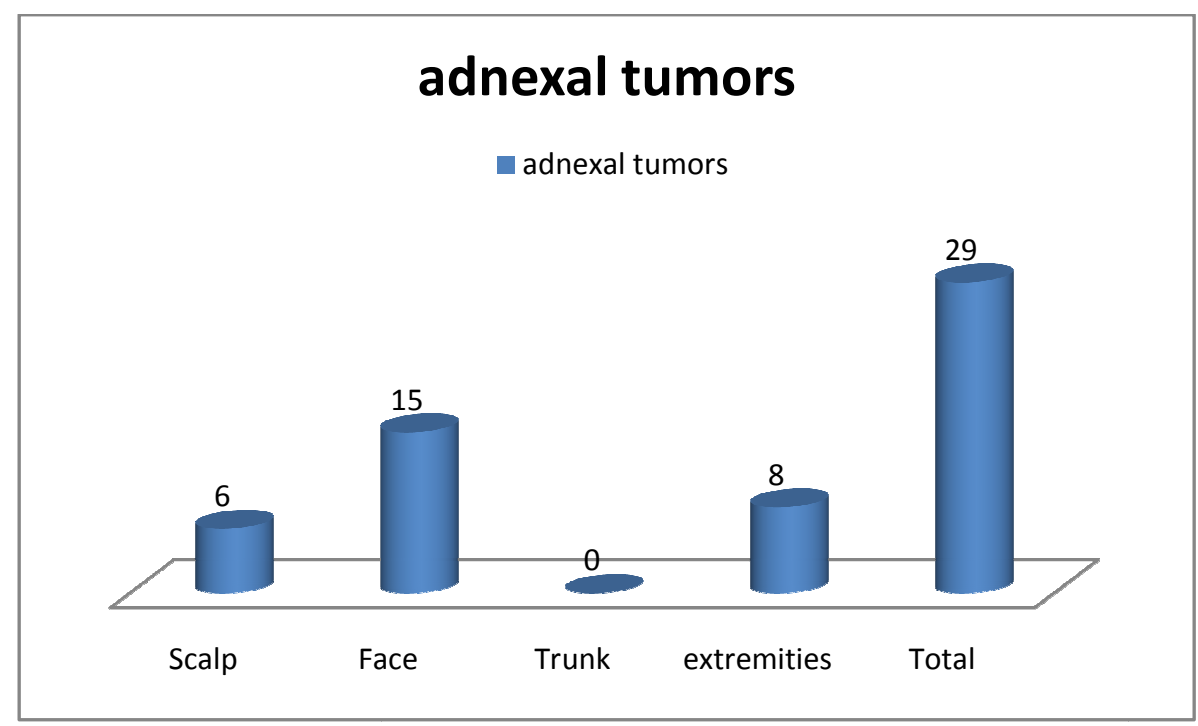

